\title{
Digitalisierung gelingt nur mit den Beschäftigten
}

\author{
Mitmachen als Schlüsselfaktor In der Einführung digitaler Technologien liegt \\ die Hoffnung, Effizienz und Effektivität von Prozessen in Krankenhäusern und \\ Pflegeeinrichtungen technikbasiert zu steigern und gleichzeitig die Arbeitsqua- \\ lität sowie die Gesundheit der Beschäftigten zu fördern. Wie können Beschäf- \\ tigte diese Digitalisierungsprozesse mitgestalten?
}

D as INQA (Initiative Neue Qualität der Arbeit) Experimentierraum Projekt untersucht und erprobt partizipative Technikeinführung unter Berücksichtigung individueller Kompetenzen und betrieblicher Organisationskultur im Krankenhaus. Für das über drei Jahren laufende Projekt DigiKIK (Digitalisierung - Krankenhaus - Interaktion - Kompetenz) wurde in vier Kliniken eine Steuerungsgruppe eingerichtet, bestehend aus den Vertretern der Berufsgruppen, Geschäftsführung und Betriebsrat. Um die Wechselwirkungen von subjektiven Kompetenzen, Nutzungserfahrungen, Nutzungspraktiken und Arbeitsbelastung in digitalisierten Arbeitskontexten zu untersuchen, wurde u.a. eine Beschäftigtenbefragung durchgeführt. Die Ergebnisse der Befragung aus dem Pflege- und Erziehungsdienst $(n=412)$ werden im
Folgenden skizziert. Insgesamt haben 1.214 Beschäftigte an der Befragung teilgenommen. Der Beitrag nimmt Bezug auf die Projekterfahrung des ersten Jahres und formuliert drei Thesen, wie Technikimplementierung positiv beeinflusst werden kann.

These 1: Individuelle Faktoren: Digitale Kompetenz als wesentliche Gelingensbedingung

Die DigiKIK Beschäftigtenbefragung zeigt, dass digitale Technik zu einem gegenwärtigen Bestandteil in den Krankenhäusern geworden ist. 90\% der Befragten nutzen digitale Technik mehrmals täglich zur Erledigung ihrer Arbeitsaufgaben. Als häufigste Nutzungsgründe wurden die Patientenversorgung, Informationsbeschaffung und Kommunikation angegeben.

\section{+ HANDLUNGSTIPPS: DAS KÖNNEN SIE TUN}

Die Projekterfahrung in DigiKIK zeigt, dass die Verbesserung von Strukturen und Abläufen nicht ohne die Beschäftigten funktionieren kann. Was können Beschäftigte selbst dafür tun, wenn sie die digitale Gestaltung der Arbeit stärker mitgestalten wollen.

Betriebliche Steuerungsgruppen einrichten: Richten Sie einen berufsgruppenübergreifenden Austausch im Rahmen von Projektsteuerungsgruppen ein. Regen Sie über den Betriebsrat, die Personalentwicklung und über das Management solche Gruppen in Ihrer Abteilung an und fragen Sie nach Möglichkeiten der berufsübergreifenden Vernetzung. Engagierte Beschäftigte können die Initiative ergreifen.

Gemeinsame Normen und Werte schaffen: Grundlegend ist die systematische Erarbeitung eines gemeinsamen Verständnisses von Digitalisierung sowie dem konkreten Nutzen einer Anwendung im Arbeitsprozess. Technik wird dann genutzt, wenn diese funktioniert und sich in den Berufsalltag sinnvoll integrieren lässt. Initiieren Sie eine Befragung dazu, verbun- den mit dem Ziel, Optimierungsvorschläge auf praktischer Ebene zu entwickeln. Die Mitgestaltung der Arbeitsabläufe ist der Faktor für eine höhere Arbeitszufriedenheit, bessere Behandlungsqualität und effizientere Prozesse.

Positive Einstellung betonen: Machen Sie deutlich, dass Sie digitalen Veränderungen grundsätzlich positiv gegenüberstehen, wenn durch die neue Technik (auch) alte Probleme beseitigt oder zumindest gelindert werden. Neue Technik kann helfen, sie muss aber passen. Ob sie passt, das können am besten die Personen bewerten, die mit dieser Technik arbeiten. Oft wird angenommen, dass Beschäftigte der Einführung von digitaler Technik kritisch gegenüberstehen. Eine völlige Fehleinschätzung, wie sich im Projekt zeigte. 


\section{PFLEGE PERSPEKTIVEN}

Die Selbsteinschätzung der hierfür notwendigen digitalen Kompetenz zeigt ein positives Bild. Die Mehrzahl (60,19\%) der befragten Personen des Pflege- und Erziehungsdienstes bewertet die eigenen digitalen Kompetenzen als „sehr gut“ bis „gut“. Nur 2,67\% gaben sich die Schulnote „mangelhaft“ oder „ungenügend“. Weiterhin stimmten 86,03\% der Befragten der Aussage zu, die digitalen Anwendungen an ihrem Arbeitsplatz ohne Probleme bedienen zu können. $82,76 \%$ gaben an mit den neuen technischen Anforderungen ihres Arbeitsbereiches gut zurechtzukommen und nur 18,78\% bereitet der Umgang mit digitaler Technik häufig Schwierigkeiten. Über die Hälfte der Befragten (62,59\%) nutzt digital aufbereitete Daten dazu, um bessere Entscheidungen an ihrem Arbeitsplatz treffen zu können.

Digitale Kompetenzen werden als individuelle Voraussetzungen für die Nutzung von Technik definiert. Im Projekt DigiKIK (digikik-projekt.de) wird „digitale Kompetenz“ nicht nur mit Anwenderkompetenz gleichgesetzt, sondern umfasst auch den sinnvollen Einsatz und Umgang mit digitalen Anwendungen - unter Berücksichtigung fachlicher Kompetenzen. Die digitalen Kompetenzen der Beschäftigten bedingen die von den Beschäftigten unterstellten Auswirkungen auf den Arbeitsplatz, Arbeitsprozesse und Belastungen und gelten als wesentliche Voraussetzung für eine erfolgreiche Technikimplementierung. Dies heißt, dass der Umgang und das Verständnis der digitalen Anwendung auf individueller Ebene dafür maßgeblich sind, ob die eingeführte Technik tatsächlich zu einer Verbesserung des Arbeitsprozesses beitragen kann.

These 2: Die Partizipation der Beschäftigten bei der Umstellung auf digitale Lösungen ist ein betrieblicher „Experimentierraum”

Die Einführung digitale Techniken soll unterstützend wirken und zu Erleichterung der Arbeit führen. Doch häufig erfolgt eine Implementierung von technischen Neuerungen in die Einrichtungen aufgrund von Gesetzesänderungen. Nur 21,08\% der Befragten ga-

Ideen einbringen: Reichen Sie eigene Ideen zur Technikgestaltung am Arbeitsplatz ein. Ermutigen Sie auch Kollegen dazu. Die Befragung zeigt, dass die Beschäftigten kompetent sind und konkrete Verbesserungsvorschläge hinsichtlich der Etablierung digitaler Technik haben. Diese Ideenbörse sollte transparent gestaltet werden, beispielsweise durch die Präsentation dieser Ideen im Rahmen eines Workshops.

Betriebliche Interessenvertretung einbinden: Reflektieren Sie Ihre eigene Rolle in Veränderungsprozessen. An welchen Prozessabschnitten hätten Sie sich gerne mehr eingebracht oder wären gern früher informiert worden? Diese Analyse kann helfen, zukünftige Prozesse besser zu planen. Um den Transfer von Management und Beschäftigten besser zu organisieren, empfiehlt sich daher die aktive Einbindung der betrieblichen Interessenvertretung. ben an, umfassend bei der Entwicklung und Bewertung von digitalen Lösungen beteiligt zu werden. Rechtzeitig vor der Einführung digitaler Technik informiert fühlten sich nur 33,16\% der Befragten. Doch ein Drittel der Befragten (30,17\%) hat selbst konkrete Ideen für den sinnvollen Einsatz weiterer digitaler Technik. Insgesamt $36,30 \%$ der Befragten würden sich gerne bei der Entwicklung und Bewertung von digitalen Lösungen stärker einbringen.

Die DigiKIK Befragungsergebnisse weisen darauf hin, dass eine umfassende Beteiligung der Beschäftigten an der Entwicklung digitaler Lösungen einen positiven Einfluss auf die körperliche und psychische Entlastung hat. 21,80\% der Befragten gaben das Vorhandensein einer solchen Beteiligung für ihr Arbeitsumfeld im Kontext der Unternehmenskultur an. 43,75\% dieser Personen fühlen sich durch den Einsatz digitaler Technik gestresst, wohingegen dies - bei den Befragten, welche keine Beteiligungsmöglichkeiten sehen - nur knapp 22,26\% angaben.

\section{These 3: Individuelle Kompetenzen und Partizipation sind eingebettet in eine betriebliche Organisationskultur}

Fest steht, dass Digitalisierung sowohl Arbeitsprozesse als auch die Organisationsstruktur und -kultur sowie die sozialen Praktiken beeinflusst. Digitalisierung determiniert hierbei nicht die Arbeitsprozesse oder Belastungen von Beschäftigten in einseitiger Richtung. Vielmehr gibt es einen wechselseitigen Zusammenhang - d.h. eine interaktive Beziehung zwischen Mensch, Technologie und Organisation. Betriebliche Digitalisierung prägt die Organisation nicht nur, sondern Organisationen haben spezifische Voraussetzungen für die Umsetzung von Digitalisierungsstrategien. Diese Voraussetzungen liegen in der jeweiligen Organisationskultur begründet. Organisationskultur umfasst gemeinsame Normen und Werte und Erfahrung. Die Kultur prägt das moralische Bewusstsein und die intrinsische Motivation und kann sich positiv oder negativ auf die Gesundheit auswirken - je nachdem wie verbindlich die Normen und Werte individuell erlebt werden und wie weit sie mit den persönlichen Überzeugen und Werten ihrer Mitglieder übereinstimmen und vereinbar sind. Digitalisierung setzt an der Organisationsstruktur an - zur erfolgreichen Implementierung sollten Struktur und Kultur zusammengedacht werden.

\section{Konstruktive Kritik willkommen}

Kernelement einer gesundheitsförderlichen Organisationskultur ist eine offene und transparente Kommunikation. So kann es gelingen, das Vertrauen der Beschäftigten in die Organisation positiv zu bestärken. 62,82\% der Befragten aus dem Pflege- und Erziehungsdienst gaben an, dass in ihrem Arbeitsbereich konstruktive Kritik und Verbesserungsvorschläge zu digitaler Technik willkommen seien ( $\mathrm{n}=174)$. Knapp 35\% dieser Personen fühlen sich durch den Einsatz digitaler Technik körperlich entlastet oder weniger gestresst. Bei den Beschäftigten, die nicht das Gefühl des Willkommenseins von konstruktiver Kritik und Verbesserungsvorschlägen erleben, empfinden nur $18 \%$ den Einsatz digitaler Technik als stressreduzierenden- oder körperlich entlastenden Faktor. Es zeigt sich demzufolge ein Unterschied zugunsten einer offenen Organisationskultur mit entsprechenden Kommunikationsstrukturen.

Der veröffentlichte psyGA Monitor „Arbeitsbezogenes Wohlbefinden" geht ebenfalls von einem entscheidenden Einfluss einer un- 


\section{PFLEGE PERSPEKTIVEN}

terstützenden Führungs- und offenen Unternehmenskultur aus. $61 \%$ der befragten Personen $(n=1.227)$ gaben bei der Frage nach „Faktoren, welche die Chance, in die Gruppe der „gesunden Engagierten“ zu gelangen, erhöhen/reduzieren“ den Faktor „Führungskraft und Unternehmenskultur" mit Abstand zu den anderen Faktoren an. Mangelnde Partizipation an Entscheidungsprozessen, wenig Selbstbestimmung bei der Arbeitsdurchführung und fehlender Handlungsspielraum sorgen für ein negatives Empfinden. Ebenso begünstigend für negative psychische Belastung am Arbeitsplatz wirken fehlendes oder negatives Feedback sowie geringe Wertschätzung und Entlohnung. Die Ergebnisse aus dem psyGA Monitor unterstreichen die Priorität einer unterstützenden Führung, offener Kommunikation, mitarbeiterorientierter Unternehmenskultur und der Weiterentwicklungsmöglichkeiten für die Beschäftigten.

\section{Offene Kommunikationskultur sorgt für Wohlbefinden}

Die Ergebnisse der DigiKIK Befragung und des psyGA Monitors stärken die Annahme des positiven Zusammenhangs zwischen einer offenen Kommunikations- und Organisationskultur und dem Wohlbefinden der Beschäftigten im Zuge von Technikeinführungen. Sowohl eine Digitalisierungsstrategie als auch das Management von Gesundheit sind Teil der Organisationsentwicklung eines Unternehmens und können in deren strategischen Leitbildern schriftlich fixiert sein. Entscheidend aber ist, ob diese Leitbilder und strategischen Ziele von den Beschäftigten gelebt werden. Wichtig dabei ist die Partizipation der Beschäftigten, die mehr ist als nur passives „Dabei-Sein“. Vielmehr umfasst der Begriff die aktive Teilhabe verbunden mit der Möglichkeit, Entscheidungen und deren Umsetzung beeinflussen zu können und wird mit „Selbstwirksamkeit“ übersetzt. DigiKIK trägt dazu bei, Partizipation im Krankenhaus aktiv zu leben und mit den Beschäftigten gemeinsam Technikeinführung so zu gestalten, dass eine Prozessoptimierung erreicht werden kann. Eine verbesserte Leistung des Systems hat letztlich einen positiven Effekt auf Organisationskultur, Wohlbefinden und Gesundheit.

\section{>II}

\section{Literatur bei der Verfasserin}

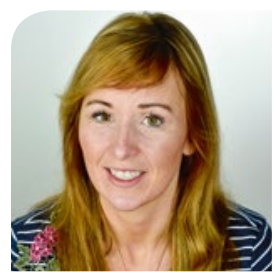

\section{Laura Schröer}

Wissenschaftliche Mitarbeiterin im Institut Arbeit und Technik, Westfälische Hochschule Gelsenkirchen Recklinghausen Bocholt, Projektleitung DigiKIK

Munscheidstraße 14, 45886 Gelsenkirchen Schroeer@iat.eu

\section{Hier steht eine Anzeige.}

\section{Springer}

